\title{
Chaotic Dynamics in Credit Constrained Emerging Economies*
}

\author{
Jordi Caballé \\ Unitat de Fonaments de l'Anàlisi Economica and CODE \\ Universitat Autònoma de Barcelona \\ Xavier Jarque \\ Departament de Matematiques \\ Universitat de Barcelona \\ Elisabetta Michetti \\ Dipartimento di Istituzioni Economiche e Finanziarie \\ Università degli Studi di Macerata
}

March 16, 2004

\begin{abstract}
A bstract
This paper analyzes the role of financial development as a source of endogenous instability in small open economies. By assuming that firms face credit constraints, our model displays a complex dynamic behavior for intermediate values of the parameter representing the level of financial development of the economy. The basic implication of our model is that economies experiencing a process of financial development are more unstable than both very underdeveloped and very developed economies. Our instability concept means that small shocks have a persistent effect on the long run behavior of the model and also that economies can exhibit cycles with a very high period or even chaotic dynamic patterns.
\end{abstract}

JEL classification codes: O16, C62.

Keywords: Chaotic Dynamics, Credit Constraints, Financial Development.

\footnotetext{
${ }^{*}$ Financial support from the Spanish Ministry of Science and Technology through grants SEC2003-306, and HI2001-0039; and from the Generalitat of Catalonia through the Barcelona Economics program of CREA and grants SGR2001-00162, SGR2001-00173, and BFM2002-01344 is gratefully acknowledged.

Correspondence address: Jordi Caballé. Universitat Autònoma de Barcelona. Departament d'Economia i d'Història Econòmica. Edifici B. 08193 Bellaterra (Barcelona). Spain. Phone: (34)-935.812.367. Fax: (34)-935.812.012. E-mail: Jordi.Caballe@uab.es
} 


\section{Introduction}

This paper considers a model where the process of financial development could be a source of endogenous instability for small open economies. Our basic macroeconomic model describes a dynamic open economy where firms face credit constraints. This means that the maximum amount entrepreneurs can borrow is proportional to the amount of their current level of wealth.

Several authors have discussed the implications of borrowing constraints on the persistence of business cycles (Bernanke and Gertler, 1989) and on the emergence of cycles in closed economies (Azariadis and Smith, 1998; and Kyotaki and Moore, 1997). Moreover, Aghion et al. (2000) showed that, when firms are credit constrained and there is debt issued both in domestic and in foreign currency, currency crises could easily arise. Later on, Aghion et al. (2001) constructed a simple monetary model where currency crises are driven by the interplay between the credit constraints of domestic firms and the existence of nominal price rigidities, which in turn leads to multiple equilibria. We will use a similar formulation, although we will focus on the real side of the economy like in Aghion et al. (1999) and Aghion et al. (2003). In particular, Aghion et al. (1999) developed a simple macroeconomic model where the combination between capital market imperfections (taking the form of borrowing constraints due to moral hazard problems) and unequal access to investment opportunities across individuals (due to the physical separation between savers and investors) generates endogenous and permanent fluctuations in aggregate GDP, investment, and interest rates. In their paper the endogenous cycles arise from two distinct factors. A larger amount invested generates more profits and more investment. However, a large investment pushes interest rates up and this reduces future profits and, thus, future investment. Finally, Aghion et al. (2003) consider a model close to ours to conclude that, at an intermediate level of financial development, economies could present unstable dynamics, while either underdeveloped or very developed ones are stable. They show that the fixed point of the entrepreneurs' wealth is stable when the level of financial development is either "high" or "low", while for some intermediate values of financial development the attractor is a two stable cycle. However, it does not follow from their work the existence of high period cycles or of chaotic dynamics.

Using bifurcation analysis we will show that economies with either very developed or very undeveloped financial markets are structurally stable, while emerging markets (with intermediate levels of financial development) are unstable in the sense that they could exhibit chaotic dynamics and, thus, the evolution of the endogenous variables of the model turns out to be unpredictable. Therefore, when the economy is going through a phase of financial development, the dynamics of the economic system could change 
dramatically and evolve from a stable fixed point to a stable cycle and, finally, to an attractor displaying aperiodic dynamic behavior.

The fluctuations displayed by the model we consider are due to two forces. First, larger investment raises both output and profits. Larger profits improve creditworthiness and raise borrowing, which in turn leads to a larger amount of investment. Simultaneously, this increase leads to a rise in the demand for the country specific input (which has constant supply) and, thus, in its relative price. This rise in input prices leads to lower profits and reduce creditworthiness, borrowing and investment, which in turn makes aggregate output fall. The conclusion is that financial development could destabilize economies exhibiting an intermediate level of financial development, which agrees with the experience documented for a number of countries. ${ }^{1}$

Other authors have investigated the possibility of chaotic dynamics in macroeconomic models by using the classical paper by Li and Yorke (1975). Thus, Stutzer (1980) and Day (1983) showed the possibility of obtaining aperiodic paths in simple models of population growth. Benhabib and Day (1981) investigated how preferences depending on past experience could lead to erratic behavior. The same authors (Benhabib and Day, 1982) have also contributed to this literature by characterizing the conditions under which chaos is obtained in overlapping generation models. Finally, Deneckere and Pelikan (1986) and Boldrin and Montrucchio (1986) analyzed the possibility of chaotic paths in neoclassical multi-sector models. Our model adds to this literature a very simple model with an imperfection in the financial market, which is a source of very complex dynamics for a wide range of parameter values.

The paper is organized as follows. In Section 2 we present the model. In Section 3 we perform the dynamic analysis in order to assess the plausibility of chaotic dynamics when entrepreneurs do not receive any exogenous income. In this case we present some general properties of the attractors of the dynamic system depending on the parameter values of the model. In Section 4 we extend the analysis to the general case with positive exogenous income and we show the global stability of either very developed or very underdeveloped financial markets. Section 5 concludes our paper. Appendix A contains some definitions concerning periodic and chaotic dynamics, while Appendix B contains the proofs of all the results of the paper.

\section{The M odel}

Let us consider a small open economy in discrete time. There are two types of individuals in this economy: the borrowers (or entrepreneurs), who own a production technology and may invest either in the production activity

\footnotetext{
${ }^{1}$ See, among many others, De Melo et al. (1985), Galvez and Tybout (1985), and Petrei and Tybout (1985).
} 
or in the international capital market, and the lenders (or families), who cannot directly invest in the production activity, but they can either lend funds to the entrepreneurs or invest in the international capital market. The international gross rate of interest is constant and equal to $r>0$.

This economy produces a unique tradeable good. The production function of this good uses capital and a country specific input (like land, real estate, or a non-tradeable natural resource), which has a constant supply equal to $Z$. Moreover, the tradeable good can be consumed or accumulated as productive capital for the following period's production. The output $y_{t}$ of the tradeable good in each period is obtained through the following Cobb-Douglas production function:

$$
y_{t}=F\left(K_{t}, z_{t}\right)=A\left(K_{t}^{\rho} z_{t}^{1-\rho}\right), \text { with } \rho \in(0,1),
$$

where $z_{t}$ is the amount of the country specific input used in period $t, K_{t}$ is the amount of capital, and $A$ is the total factor productivity. We assume that $A>r$, since the entrepreneurs would do not find profitable to invest in the production activity otherwise. We assume that capital fully depreciates after one period.

The total investment $I_{t}$ in period $t$ is devoted to purchase both capital and country specific input. For a given level of investment, the optimal demand $z_{t}$ for the country specific input in each period $t$ arises from the maximization of the profit function subject to the budget constraint

$$
I_{t}=K_{t}+p_{t} z_{t}
$$

where $p_{t}$ is the price of the country specific input expressed in units of the tradeable good. The first order condition of the previous problem yields

$$
z_{t}=\left(\frac{1-\rho}{p_{t}}\right) I_{t}
$$

Therefore, the country specific input equilibrium price is obtained by equating the country specific input equilibrium demand (2) with its constant supply $Z$,

$$
p_{t}=\left(\frac{1-\rho}{Z}\right) I_{t}
$$

Finally, substituting (2) into (1), we may write the total equilibrium output $y_{t}$ in terms of the level of investment $I_{t}$ and the price $p_{t}$,

$$
y_{t}=G\left(p_{t}\right) I_{t}, \quad \text { with } \quad G\left(p_{t}\right)=\frac{A \rho^{\rho}(1-\rho)^{1-\rho}}{p_{t}^{1-\rho}} .
$$

Note that $G\left(p_{t}\right)$ can be viewed as the gross return of a unit of investment. 
We assume that the credit market operates imperfectly due to, say, either adverse selection or moral hazard considerations. In particular, we assume that an entrepreneur with wealth $W_{t}$ may borrow at most the amount $L_{t}$, with $L_{t} \leq \mu W_{t}$, as the entrepreneur's wealth serves as a collateral for the loan. ${ }^{2}$ Therefore, the investment in each period is bounded above by $(1+\mu) W_{t}$. The proportional coefficient $\mu \geq 0$ can be viewed as a credit multiplier reflecting the level of financial development of the domestic economy.

The dynamics of the model is described as follows. In period $t$ entrepreneurs decide the amount of borrowing (and, thus, of investment), and pay the cost of the country specific input. Hence, at period $t+1$ the entrepreneurs receive the corresponding profits and pay the cost of debt $r L_{t}$. We assume here that entrepreneurs save a constant fraction $(1-\alpha)$ of their total wealth at the end of each period, where $\alpha$ is the constant propensity to consume. ${ }^{3}$ Therefore, the dynamics of the entrepreneurs' wealth is given by

$$
W_{t+1}=(1-\alpha)\left(e+y_{t}-r L_{t}\right),
$$

where $e \geq 0$ is an exogenous income in terms of tradeable good and $L_{t} \leq \mu W_{t}$.

Let us consider first the case where $G\left(p_{t}\right) \geq r$, which means that the productive investment return exceeds the international capital market return and, hence, the entrepreneurs will invest in the productive project the largest amount they can borrow,

$$
I_{t}=(1+\mu) W_{t} .
$$

Total output is now given by

$$
y_{t}=G\left(p_{t}\right)(1+\mu) W_{t} .
$$

Substituting (3) and (6) in the previous equation, we obtain the total output in period $t$ as a function of wealth,

$$
y_{t}=\xi W_{t}^{\rho}, \quad \text { with } \xi=A \rho^{\rho}(1+\mu)^{\rho} Z^{1-\rho} .
$$

As follows from (5), the dynamics of entrepreneurs' wealth is

$$
W_{t+1}=(1-\alpha)\left(e+y_{t}-r \mu W_{t}\right)
$$

which, after using (7) becomes

$$
W_{t+1}=(1-\alpha) W^{\rho}\left(\xi-r \mu W^{1-\rho}\right)+(1-\alpha) e .
$$

\footnotetext{
${ }^{2}$ This is the type of constraint found in Bernanke and Gertler (1989).

${ }^{3}$ This simple saving rule could be derived under the assumption that entrepreneurs maximize the discounted sum of instantaneous utilities when these utilities are logarithmic (see Woodford, 2003).
} 
The previous equation describes the dynamics of the entrepreneurs' wealth as long as

$$
G\left(p_{t}\right) \geq r .
$$

Combining (3), (4), and (6), the previous inequality holds whenever $W \leq W^{m}$, where the critical point $W^{m}$ is given by

$$
W^{m}=\left(\frac{A}{r}\right)^{\frac{1}{1-\rho}}\left(\frac{Z}{1+\mu}\right) \rho^{\frac{\rho}{1-\rho}}
$$

As it can be immediately seen from (9), the impact of a change in the volume of current wealth could be ambiguous. This is so because, even if an increase in wealth raises the investment, the amount of invested wealth depends negatively on the price $p$ of the country specific input, and this price depends positively on current wealth. Hence, two distinct effects coexist: a positive wealth effect on investment for a given price $p$ and a negative price effect via a greater demand for the country specific input.

Let us now consider the case where $G\left(p_{t}\right)<r$. In this case the entrepreneurs have no incentives to borrow up to the credit constraint because the return from the productive investment is lower than the return from the international capital market. Hence, they borrow until the point where the productive investment return is equal to the international capital market return, that is, until $y_{t}-r L_{t}=r W_{t}$. Therefore, substituting the previous equation into (5), we find the dynamic equation for the wealth when $W>W^{m}$,

$$
W_{t+1}=(1-\alpha)\left(e+r W_{t}\right) .
$$

The asymptotic behavior of wealth is thus determined by the iterates of the following function (see (9) and (11)):

$$
f\left(W_{t}\right)= \begin{cases}(1-\alpha)\left[e+W_{t}^{\rho}\left(\xi-r \mu W_{t}^{1-\rho}\right)\right] \equiv f^{l}\left(W_{t}\right), & \text { if } 0 \leq W_{t} \leq W^{m}, \\ (1-\alpha)\left(e+r W_{t}\right) \equiv f^{r}\left(W_{t}\right), & \text { if } W_{t}>W^{m} .\end{cases}
$$

The iterates of $f$ describe a one-dimensional discrete dynamical system. In the next sections we will analyze the general dynamic properties of the system depending on the parameters of the model and we will determine the cases where complex (or even chaotic) dynamics could appear. Note that the dynamic behavior of the other endogenous variables of the model, output $y_{t}$, investment $I_{t}$, and price of the country specific input $p_{t}$, is entirely determined by the behavior of the entrepreneurs' wealth, as follows immediately from the previous analysis. 
It is straightforward to see that, for any value of the parameters, $f^{l}\left(W_{t}\right)$ is a single peaked function with a maximum at

$$
W^{M}=\left(\frac{r \mu}{\xi \rho}\right)^{\frac{1}{\rho-1}}
$$

while $f^{r}\left(W_{t}\right)$ is linear. We notice, however, that $f\left(W_{t}\right)$ has a local maximum at $W^{M}$ only if $W^{M}<W^{m}$.

\section{Dynamical A nalysis W ithout Exogenous Income}

In this section we describe the long run dynamics of the model when the exogenous income $e$ is equal to zero. This analysis will be helpful in the next section, where we study the case with positive exogenous income, $e>0$. For the rest of the paper we will assume the empirically plausible inequality $(1-\alpha) r<1$, which ensures the existence a positive fixed point for the function $f$.

When $e=0$ it is immediate to see that $f$ has a repelling fixed point at $W=0$. Other dynamic properties are summarized in the following lemma:

Lemma 3.1. (a) If $\mu \in\left[0, \frac{\rho}{1-\rho}\right)$, then $W^{M}>W^{m}$ and $f$ is a strictly increasing function. Moreover $f$ has a unique positive, globally stable fixed point $W^{\star} \in\left(0, W^{m}\right)$.

(b) If $\mu \in\left[\frac{\rho}{1-\rho}, \frac{\rho}{r(1-\alpha)(1-\rho)}\right)$, then $W^{M} \leq W^{m}$ and $f$ has a unique positive, globally stable fixed point $W^{\star} \in\left(0, W^{M}\right)$.

(c) If $\mu \in\left[\frac{\rho}{(1-\alpha) r(1-\rho)}, \infty\right)$, then $f$ has a unique positive fixed point $W^{\star} \in\left[W^{M}, W^{m}\right)$ and $f^{\prime}\left(W^{\star}\right) \leq 0$.

From the previous lemma we conclude that, in order to obtain a chaotic dynamics for the entrepreneurs' wealth, the parameter value of $\mu$ must be sufficiently high. In particular, we must have $\mu \geq \frac{\rho}{(1-\alpha) r(1-\rho)}$. Moreover, the previous lemma states the global stability for economies with low values of $\mu$, that is, for economies exhibiting a low level of financial development. As we will see in Section 4, this result also holds in the case of positive exogenous income.

Next we study the first period bifurcation of our model.

\section{Lemma 3.2.}

(a) If $\mu \in\left[\frac{\rho}{r(1-\alpha)(1-\rho)}, \frac{1+\rho}{r(1-\alpha)(1-\rho)}\right)$, then the fixed point $W^{\star}$ is the unique positive globally stable fixed point of $f$. 
(b) A 2-period bifurcation occurs at $\mu_{2}=\frac{1+\rho}{r(1-\alpha)(1-\rho)}$ and the derivative of $f$ at $W^{\star}$ is -1 . Hence, a 2-period globally stable cycle exists if $\mu \in\left(\mu_{2}, \mu_{2}+\delta\right)$ for $\delta$ sufficiently small.

Now we provide an example illustrating the asymptotic dynamics of wealth as a function of the degree of financial development, which is parametrized by the parameter $\mu$. Thus, a high value of $\mu$ corresponds to a financially developed economy, while low values are associated to financial underdevelopment.

The approach we consider is mainly numerical but allows us to provide a complete description of the dynamics of the model when $e=0$. The main results of our analysis for this case are the following:

1. For small values of the parameter $\mu$, the asymptotic dynamics is simple and governed by a low (1 or 2 ) period global attracting cycle. Thus, economies are stable in the sense that we may predict their evolution in the long run. Moreover, we also have structural stability of the asymptotic equilibrium as the globally attracting cycle remains under small perturbations of the parameter value $\mu$.

2. For large values of $\mu$, the asymptotic dynamics can be either predictable (although the global attracting cycle can be of an arbitrarily large period), or chaotic (and, thus, unpredictable).

As we will see, result 1 will still hold for the general case where $e>0$. However, result 2 does not hold, since we will show that not only economies with a low value of $\mu$ are stables but also financially developed economies are.

For the numerical analysis we consider the empirically plausible parameter values $\rho=1 / 3, \alpha=0.8, r=1.02$. Moreover we also consider the arbitrary parameter values $A=3 / 2$ and $Z=100$. We will perform a robustness analysis, which will allow us to see that the qualitative properties of the dynamics of $W_{t}$ remain for different values of $A$ and $Z$.

Under our parameter values and $e=0$ the function $f$ becomes

$f= \begin{cases}\left(W_{t}\right)^{\frac{1}{3}}\left[\sqrt[3]{900}(1+\mu)-\frac{51}{250}\left(W_{t}\right)^{\frac{2}{3}} \mu\right] \equiv f^{l}\left(W_{t}\right), & \text { for } 0 \leq W \leq W^{m} \\ \frac{51}{250} W \equiv f^{r}\left(W_{t}\right), & \text { for } W>W^{m},\end{cases}$

where $W^{m}=\frac{12500}{17 \sqrt{51}(1+\mu)}$.

Notice that $f^{r}$ is a linear function whose slope does not depend on $\mu$. On the other hand $f^{l}$ is a single peaked concave function with the local 
maximum at the point

$$
W^{M}=\frac{12500(1+\mu)^{1 / 2}}{153 \sqrt{17} \mu^{3 / 2}} .
$$

The asymptotic dynamics described by $f$ has to be studied depending on the relative position of $W^{m}, f\left(W^{m}\right), W^{M}$ and $f\left(W^{M}\right)$. We will first perform our analysis for relatively small values of $\mu$ (for which the dynamics is simple) and then we consider relatively large values of $\mu$ (for which the dynamics becomes complex).

For positive values of $\mu$ smaller than 2.451, the dynamics of $f$ is trivial, since the stable steady state of wealth belongs to the interval where $f^{l}$ is increasing. Moreover, for $\mu \in(0,0.5]$ the steady state $W^{*}$ lies in the interval $\left(0, W^{m}\right]$, whereas $W^{*} \in\left(0, W^{M}\right]$ for $\mu \in(0.5,2.451]$. Therefore, the path of wealth is globally stable and monotonic around the steady state $W^{*}$ (see Panel (a) of Figure 1). For values of $\mu$ larger than 2.451, the attracting fixed point $W^{\star}$ belongs always to the decreasing part of $f^{l}$ and $f^{\prime}\left(W^{\star}\right)=\frac{1}{3}-\frac{17 \mu}{125}$. Therefore, for $\mu \in(2.451,9.804)$, the path of $W_{t}$ is globally stable and the convergence is oscillating around the steady state (see Panel (b) of Figure 1). When $\mu=9.804$ we obtain the first period doubling bifurcation and the resulting attracting cycle has period 2 and belongs to the interval $\left(W^{M}, f\left(W^{M}\right)\right)$. The pair $\left(W^{1}, W^{2}\right)$ gives the two points of the 2-period cycle. Notice that when $\mu \in(9.804,11.203)$ it holds that $W^{M}<W^{1}<W^{2}<W^{m}$ (see Panel (c) of Figure 1). At $\mu=11.203$ we have $f\left(W^{M}\right)=W^{m}$, so that when $\mu \in(11.203,25.239)$ the attracting 2-period cycle $\left(W^{1}, W^{2}\right)$ lies in the invariant set $\left(W^{M}, f\left(W^{M}\right)\right)$ and satisfies $W^{M}<W^{1}<W^{m}<W^{2}$ as $f\left(W^{m}\right)<W^{M}$ and $f\left(W^{M}\right)>W^{m}$ for those values of $\mu$ (see Panel (d) of Figure 1).

\section{[Insert Figure 1]}

The previous analysis shows that the interval $\mu \in[0,25.239)$ gives raise to simple dynamics, where the entrepreneurs' wealth is asymptotically given by a stable fixed point or a 2-period stable cycle. Therefore, in this case we are facing a predictable and structurally stable economy in the long run. In fact, the dynamics of wealth for those values of the parameter $\mu$ agrees with our Lemmae 3.1 and 3.2, since $\frac{\rho}{1+\rho}=0.5, \frac{\rho}{r(1-\alpha)(1-\rho)}=2.451$, and $\frac{1+\rho}{r(1-\alpha)(1-\rho)}=9.804$. As we will show in Section 4 , this simple dynamics still holds when $e>0$.

A more complex dynamics appears when $\mu$ increases above the value 25.239. When $\mu=25.239$ we get $f\left(W^{m}\right)=W^{M}$ and $W^{m}<f\left(W^{M}\right)$. As the value of $\mu$ increases, the asymptotic dynamics belongs to the interval $\left[f\left(W^{m}\right), f^{2}\left(W^{m}\right)\right]$. Moreover, at the value $\mu=25.239$, there is an attracting 2-period cycle with $W^{M}<W^{1}<W^{m}<W^{2}<f^{2}\left(W^{m}\right)$. 
In order to study the case of those more financially developed economies in a simpler manner, we first compose the function

$$
f:\left[f\left(W^{m}\right), f^{2}\left(W^{m}\right)\right] \rightarrow\left[W^{m}, f^{2}\left(W^{m}\right)\right]
$$

with an homeomorphism $h$ so that $g=h\left(f\left(h^{-1}\right)\right)$ is a function from [0,1] to $[0,1] .{ }^{4}$ Obviously, the dynamics associated with $f$ and $g$ display the same qualitative properties. The advantage of working with the function $g$ instead of $f$ lies on the fact that the windows where the asymptotic dynamics occurs is $[0,1]$, which is independent of $\mu$. Therefore, the analysis of the comparative statics for the different values of $\mu$ becomes much easier. Note that when $\mu>25.239$ the continuous function $g$ maps the interval $[0,1]$ into itself and the steady state is unstable, as follows from Lemma 3.2. Therefore, wealth never grows without bound nor converges to a steady state (for almost all initial conditions). Hence, it must fluctuate forever, either converging to a cycle or following an aperiodic path (see Definition 6 in Appendix A). We next investigate under which circumstances the attracting set $[0,1]$ contains attracting high-period cycles or becomes a chaotic attractor.

If $g^{l}$ and $g^{r}$ denote the two components of $g$ corresponding to $f^{l}$ and $f^{r}$, respectively, we get after some computations that

$$
\begin{aligned}
& g^{l}\left(W_{t}\right)=\frac{\delta_{\mu} W_{t}-25 \mu\left(510+51(-10+\sqrt[3]{204}) W_{t}\right)-62500+1250 \sqrt[3]{102\left(250+\beta_{\mu} W_{t}\right)}(1+\mu)}{250 \beta_{\mu}}, \\
& g^{r}\left(W_{t}\right)=\frac{-49750}{250 \beta_{\mu}}+0.204 W_{t} \\
& W^{m}=\frac{49750}{51 \beta_{\mu}}, \text { with } \\
& \beta_{\mu}=-250+25 \sqrt[3]{204}+(-51+25 \sqrt[3]{204}) \mu \text { and } \\
& \delta_{\mu}=-51(-51+25 \sqrt[3]{204}) \mu^{2} .
\end{aligned}
$$

Note that $g^{r}$ is a linear function with a constant slope, $g^{l}$ is a monotone decreasing function and $W^{m} \rightarrow 0$ as $\mu \rightarrow \infty$. We plot the function $g$ in Figure 2.

$$
\text { [Insert Figure 2] }
$$

In what follows we will show that, as we increase the value of the parameter $\mu$, dynamic complexity will arise. Recall that the multiplier $\varrho$ of a $n$-period cycle is

$$
\varrho=\prod_{i=1}^{n} f^{\prime}\left(W^{i}\right),
$$

\footnotetext{
${ }^{4}$ The function $h$ is simply a linear transformation depending on $\mu$ that assigns the value 0 to $f\left(W^{m}\right)$ and the value 1 to $f^{2}\left(W^{m}\right)$ for each $\mu$.
} 
where $\left\{W^{i}\right\}_{i=1}^{n}$ are the points of the $n$-period cycle. It can be seen from the function $g$ that the attracting 2-period cycle $\left(W^{1}, W^{2}\right)$ obtained at $\mu=25.239$ remains for a while until we arrive at the new period doubling bifurcation. Panel (a) of Figure 3 displays the 2-period cycle for $\mu=28$. The doubling bifurcation occurs when the multiplier $\varrho$ of the 2-period attracting cycle is -1 , that is, when $\mu=48.055$. After this bifurcation the asymptotic dynamics is given by an attracting 4-period cycle with positive multiplier and two points lying in the interval $\left(0, W^{m}\right)$ and two points lying in the interval $\left(W^{m}, 1\right)$. Panel (b) of Figure 3 displays a 4-period cycle for $\mu=50$.

[Insert Figure 3]

At $\mu=53.953$ one of the points of the 4-period cycle is the critical point $W^{m}$. The presence of the non-differentiable point $W^{m}$ makes this bifurcation atypical (it is called a "border collision bifurcation"). However, applying Corollary 3 in Nusse and Yorke (1995), it can be proved that a bifurcation from a 4-period cycle to a 8-period cycle occurs at this value of $\mu$.

When $\mu=54.925$ the critical point $W^{m}$ of the function $f$ is preperiodic (see Definition 5 in Appendix A) as $f\left(W^{m}\right)=0, f(0)=1$, and $f(1)=$ $W^{\star} .{ }^{5}$ Thus, for this value of $\mu$, no attracting cycles exist, since the basin of attraction of these cycles cannot contain the critical point $W^{m}$ (see Proposition 2 in Nusse and Yorke, 1995). Consequently, the set of aperiodic paths under the map $g$ has full Lebesgue measure in $[0,1]$, which means that the interval $[0,1]$ is a chaotic attractor under $g$ (see Definition 9 in Appendix A) ${ }^{6}$ Note that the fact that the interval $[0,1]$ is a chaotic attractor under $g$ implies immediately that the chaotic attractor under the original map $f$ has positive Lebesgue measure when $\mu=54.925$.

Finally, when $\mu=60.936$ the image of $W=1$ is equal to $W^{m}$ and, hence, we obtain a 3-period cycle. This parameter value is the initial point of an interval for which an attracting 3-period cycle exists (see the Panel (a) of the bifurcation diagram shown in Figure 4).

\section{[Insert Figure 4]}

The existence of two distinct values of $\mu$, one giving raise to chaotic dynamics and the other generating simple stable dynamics repeats over and over as $\mu$ increases, as the following proposition confirms:

Proposition 3.3. For any integer $k>0$, there exist a value $\mu_{k}$ of the parameter $\mu$ such that the corresponding critical point $W_{\mu_{k}}^{m}$ of the function

\footnotetext{
${ }^{5}$ A preperiodic point is usually called a Misiurewicz point.

${ }^{6}$ Moreover, the topological entropy of the map $f$ for $\mu=54.925$ is positive, which confirms the existence of a chaotic attractor (see Corollary 4.4.9 in Alsedà et al., 2001). Recall that the topological entropy is given by $\lim _{n \rightarrow \infty} \frac{\ln N_{n}}{n}$ where $N_{n}$ is the number of distinct $n$-period cycles.
} 
$f$ is a $(k+2)$-periodic point. Moreover the sequence $\left\{\mu_{k}\right\}$ is monotonically increasing in $k$ and $\mu_{k} \rightarrow \infty$ as $k \rightarrow \infty$.

The next proposition shows the existence of a special preperiodic point leading at a $(k+2)$-period cycle for all $k>0$.

Proposition 3.4. For any integer $k>0$, there exist a value $\mu_{k}^{\prime}$ of the parameter $\mu$ such that the critical point $W_{\mu_{k}^{\prime}}^{m}$ of the function $f$ is preperiodic and its preperiod is $k+2$. Moreover the sequence $\left\{\mu_{k}^{\prime}\right\}$ is monotonically increasing in $k$ and $\mu_{k}^{\prime} \rightarrow \infty$ as $k \rightarrow \infty$.

In our example, the first values of the two sequences $\left\{\mu_{k}\right\}$ and $\left\{\mu_{k}^{\prime}\right\}$ are

$$
\begin{aligned}
& \mu_{1}=60.936, \mu_{2}=304.676, \mu_{3}=1499.48, \mu_{4}=7356.363 \\
& \mu_{1}^{\prime}=54.925, \mu_{2}^{\prime}=298.793, \mu_{3}^{\prime}=1493.594, \mu_{4}^{\prime}=7350.175
\end{aligned}
$$

We show in Figure 4 the bifurcation diagrams as $\mu$ increases. The numerical experiments illustrate our previous statements. First, we observe large intervals for the values of $\mu$ where the dynamics only bifurcates from stable cycles of period $k$ to stable cycles of period of period $2 k$. In these intervals the economy is predictable and structurally stable. Second, we observe values of $\mu$ where the dynamics is extremely complex and the economy is unpredictable. Third, based on the previous two propositions, this pattern repeats over and over as $\mu$ increases and, hence, there is not a value of $\mu$ above which stability holds in the long run. As we will prove in the following section, this last result does not longer hold for the case $e>0$.

Recall that the parameters values we have been using for the total factor productivity $A$ and for the supply of the country specific input $Z$ are arbitrary. Here we want to show by means of numerical simulations that they do not affect the qualitative dynamics of the model but only the quantitative ones. In Figures 5(a) and 5(b), we present the bifurcation diagrams when $A=20$ or $Z=300$, respectively. In these simulations we are using the original function $f$ because it allow us to show that the dynamics is quantitatively different, since the amplitude of the invariant set where the asymptotic dynamics lies is changing. Moreover, we can observe that the qualitative dynamics remains unchanged: we have the same bifurcations at the same values of $\mu$ we presented in Figure 4 and, thus, all the previous results still hold. This fact is confirmed if we look at Figures 6(a) and 6(b), where we can see how the dynamics change when both the parameters $\mu$ and $A$ or $\mu$ and $Z$ change. We assign a different shading to each region in the $(A, \mu)$ and $(Z, \mu)$ planes, according to the type of attractor of the map $f$. The different regions are separated by straight horizontal lines, which means that the choice of the values for $A$ or $Z$ does not affect qualitatively the asymptotic dynamics of the model.

[Insert Figures 5 and 6 ] 


\section{Dynamic Analysis with Positive Exogenous Income.}

Let us now study the dynamics of the model in the case with positive exogenous income, that is, when $e>0$. The resulting dynamics is now determined by the function (12). Obviously, the values of the critical point $W^{m}$ and of the maximum $W^{M}$ are the same as when $e=0$ (see (10) and (13)). Panel (a) of Figure 7 illustrates the changes of the function $f$ as $e$ increases. However, both the existence and the qualitative properties of the attracting sets are affected by the fact that $e>0$. Although for this case it is not longer possible to obtain explicit expressions neither for the fixed point nor for its derivatives, we may use the computations of Section 3 to show the existence and uniqueness of global attractors in this new setting.

[Insert Figure 7]

The main result of this section is that, for any positive exogenous income $e$, we have stable dynamics for $W_{t}$ (that is, a globally stable fixed point) for low as well as for high levels of financial development. Moreover, for any positive $e$, there are intermediate levels of financial development (that is, a bounded interval of values of $\mu$ ) for which the dynamics would be complex or even chaotic.

Proposition 4.1. Let $e>0$.

(a) $W=0$ is not a fixed point.

(b) The function $f\left(W_{t}\right)$ has a unique positive, globally stable fixed point $W^{\star}$ for each $\mu \in\left[0, \mu^{m}\right)$, where $\mu^{m} \equiv \frac{\rho}{(1-\rho)}$.

(c) The function $f\left(W_{t}\right)$ has a globally stable fixed point $W^{\star}$ with $W^{\star} \geq W^{m}$ for each $\mu \in\left[\mu^{M}(e), \infty\right)$, where

$$
\mu^{M}(e) \equiv\left(\frac{A}{r}\right)^{\frac{1}{1-\rho}} Z \rho^{\frac{\rho}{1-\rho}} \frac{1-(1-\alpha) r}{(1-\alpha) e}-1 .
$$

The previous proposition shows that, for each positive value of $e$, there exists a large enough value $\mu^{M}(e)$ of the parameter $\mu$ guaranteeing the stability of financially developed economies. Intuitively, this is due to the fact that as $\mu \rightarrow \infty$ the critical point $W^{m}$ tends to $(1-\alpha) e$. The latter limit point is positive so that it has to pass from the right to the left of the $45^{\circ}$-degree line of the Panel (b) in Figure 7. Therefore, the stability of financially developed economies is obtained if and only if $e$ is positive. 
Note in this respect that global stability does not hold for $e=0$ as was established in Lemma 3.3.

Panel (a) of Figure 8 shows the bifurcation diagram for the parameter values of our benchmark economy with $e=4$. Moreover, we should emphasize that $\mu^{M}(e)$ is a decreasing function of $e$, as is shown in Panel (b) of Figure 8. The global picture is thus as follows. Let us fix $e$ and, then, for small values of $\mu\left(\mu<\mu^{m}\right)$ we have a globally stable fixed point lying below $W^{m}$ and, for high values of $\mu$ (i.e., when $\left.\mu \geq \mu^{M}(e)\right)$ we have also a globally stable fixed point lying above $W^{m}$. For intermediate values of financial development we may found complex or even chaotic dynamics. As follows from Propositions 3.3 and 3.4, the potential dynamic complexity depends on how many terms of the sequences $\left\{\mu_{k}\right\}$ or $\left\{\mu_{k}^{\prime}\right\}$ are smaller than $\mu^{M}(e)$. In particular, for high values of $e$ the value of $\mu^{M}(e)$ is so small that the attracting set is given by either a fixed point or a low period cycle, while for small values of $e$ the value of $\mu^{M}(e)$ is large enough so that emerging economies admit chaotic behavior.

\section{[Insert Figure 8]}

To understand the resulting dynamics of our model note that, on the one hand, for low values of $\mu$ the credit constraint faced by entrepreneurs is so strong that investment is insensitive to the amount of current wealth. On the other hand, for very high values of $\mu$ the amount of current wealth is also irrelevant for investment since entrepreneurs are not credit constrained. Only for intermediate values of the parameter representing the degree of financial development, the current level of investible funds are relevant for the determination of investment and, thus, for future wealth.

Note that in the chaotic region the path of the entrepreneur's wealth is sensitive to the initial conditions, as is implied by the aperiodicity of the dynamics. Therefore, small transitory shocks end up having permanent effects in the long run.

While Proposition 4.1 establishes the maximum value of $\mu$ such that the economy is stable for each choice of $e>0$, the following proposition defines a minimum value of $e$ such that the economy is stable for every $\mu \geq 0$.

Proposition 4.2. Let $\mu \geq 0$. The function $f\left(W_{t}\right)$ has a globally stable fixed point $W^{\star}$ for each $e>e^{\star}$, where

$$
e^{\star} \equiv \frac{1-(1-\alpha) r}{1-\alpha}\left(\frac{A}{r}\right)^{\frac{1}{1-\rho}} Z \rho^{\frac{\rho}{1-\rho}} .
$$

The previous proposition shows that the greater is the exogenous income, the more likely is that the economy be stable. 


\section{Conclusions}

We have studied the asymptotic dynamics of entrepreneurs' wealth in a small open economy with credit constrains under a Cobb-Douglas technology. A similar model has been considered in Aghion et al. (2003) using a Leontieff technology. These authors only showed that financial underdeveloped, as well as very developed economies, present stable fixed points, whereas intermediate levels of financial development could be a source of instability. They obtain the instability result by showing that, for some range of parameter values, a 2-period cycle appears.

Our analysis differs from that of Aghion et al. (2003) in the following aspects:

- We show that financially developed economies present complex dynamics when there is no exogenous income of the tradeable good. However, very developed economies are stable when entrepreneurs have positive exogenous income;

- We derive sufficient conditions on the parameter values of the model in order to obtain global stability;

- We show that economies with an intermediate level of financial development could present dynamics more complex than a 2-period cycle, since they could display cycles with a high period or even chaotic dynamics. Those cycles are robust as they remain under perturbations of the parameter $\mu$ representing the level of financial development. In this case, even though the dynamics becomes complex, it is still predictable. This predictability does not longer hold in the chaotic region. Moreover, when the economy displays chaotic dynamics, small temporary shocks turn out to have permanent effects.

Note that when there is no exogenous income, the complex dynamics occurs for arbitrarily large values of the parameter $\mu$. However, the range of values of $\mu$ for which complexity arises is bounded when the exogenous income is positive. Thus, in such a case, there is a sufficiently high level of financial development that guarantees stability in the long run. 


\section{References}

[1] Alsedà, L., J. Llibre and M. Misiurewicz (2001), Combinatorial Dynamics and Entropy in Dimension One, World Scientific. Singapore.

[2] Aghion, P., P. Bacchetta, and A. Banerjee (2000), A Simple Model of Monetary Policy and Currency Crises, European Economic Review 44, 728-738.

[3] Aghion, P., P. Bacchetta, and A. Banerjee (2001), Currency Crises and Monetary Policy in an Economy with Credit Constraints, European Economic Review 45, 1121-1150.

[4] Aghion, P., P. Bacchetta, and A. Banerjee (2003), Financial Development and the Instability of Open Economies, Journal of Monetary Economics (forthcoming).

[5] Aghion, P., A. Banerjee, and T. Piketty (1999), Dualism and Macroeconomic Volatility, Quarterly Journal of Economics 114, 13571397.

[6] Azariadis, C. and B. Smith (1998), Financial Intermediation and Regime Switching in Business Cycles, American Economic Review 88, $516-536$.

[7] Benhabib, J. and R. H. Day (1981), Rational Choice and Erratic Behaviour, Review of Economic Studies 48, 459-471.

[8] Benhabib, J. and R. H. Day (1982), A Characterization of Erratic Dynamics in the Overlapping Generations Model, Journal of Economic Dynamics and Control 4, 37-55.

[9] Bernanke, B. and M. Gertler (1989), Agency Costs, Net Worth, and Business Fluctuations, American Economic Review 79, 14-31.

[10] Boldrin, M. and L. Montrucchio (1986), On the Indeterminacy of Capital Accumulation Paths, Journal of Economic Theory 40, 26-39.

[11] Day, R. H. (1982), Irregular Growth Cycles, American Economic Review 72, 406-414.

[12] De Melo, J., R. Pascale, and J. Tybout (1985), Microeconomic Adjustments in Uruguay during 1973-81: The Interplay of Real and Financial Shocks, World Development 13, 965-1015.

[13] Deneckere, R. and S. Pelikan (1986), Competitive Chaos, Journal of Economic Theory 40, 13-25. 
[14] Devaney, R.L. (1992), A First Course in Chaotic Dynamical Systems, Addison Wesley.

[15] Galvez, J. and J. Tybout (1985), Microeconomic Adjustments in Chile during 1977-81: The Importance of Being a Grupo, World Development 13, 969-994.

[16] Kyotaki, N. and J. Moore (1997), Credit Cycles, Journal of Political Economy 105, 211-248.

[17] Li, T.Y. and J. A. Yorke (1975), Period Three Implies Chaos, American Mathematical Monthly 82, 895-992.

[18] Nusse, H. E. and J. A. Yorke (1995), Border-collision Bifurcations for Piecewise Smooth One-dimensional Maps, International Journal of Bifurcation and Chaos 5-1, 189-207.

[19] Petrei, A. H. and J. Tybout (1985), Microeconomic Adjustments in Argentina during 1976-81: The Importance of Changing Levels of Financial Subsidies, World Development 13, 949-968.

[20] Stutzer, M. J. (1980), Chaotic Dynamics and Bifurcation in a Macro Model, Journal of Economic Dynamics and Control 2, 353-376.

[21] Woodford, M. (2003), Imperfect Financial Intermediation and Complex Dynamics, in S. Zamagni and E. Agliardi, eds., Time in Economic Theory, Edward Elgar. 


\section{Appendix}

\section{A Definitions}

Definition 1. A path associated with (or under) the real-valued map $f: B \longrightarrow B$ is a sequence $\left\{W_{t}\right\}_{t=0}^{\infty}$ such that $W_{t+1}=f\left(W_{t}\right)$, for $t=0,1,2 \ldots$

Definition 2. The iterated map $f^{n}$ is defined recursively by $f^{0}(W)=W$ and $f^{n}(W)=f\left(f^{n-1}(W)\right)$ for $n=1,2, \ldots$

Definition 3. A $n$-period cycle is a vector $\left(W^{1}, W^{2}, \ldots, W^{n}\right)$ such that $f^{n}\left(W^{i}\right)=W^{i}$ and $f^{k}\left(W^{i}\right) \neq W^{i}$ for $1 \leq k<n$, for all $i=1,2, \ldots, n$. The elements $W^{i}, i=1,2, \ldots, n$, of the $n$-period cycle are termed $n$-periodic points. A 1-periodic point is termed steady-state or fixed point of $f$. A $n$-periodic path is a path under $f$ that contains only $n$-periodic points.

Definition 4 . The point $W^{p}$ is asymptotically $n$-periodic if there exists a $n$-periodic point $W^{i} \neq W^{p}$ such that

$$
\lim _{m \rightarrow \infty}\left[f^{m}\left(W^{p}\right)-f^{m}\left(W^{i}\right)\right]=0 .
$$

A path $\left\{W_{t}\right\}_{t=0}^{\infty}$ associated with the real-valued map $f: B \longrightarrow B$ is asymptotically $n$-periodic if it contains only asymptotically $n$-periodic points.

Definition 5. The point $W^{p}$ is called preperiodic if there exists a finite positive integer $m$ and a periodic point $W^{i} \neq W^{p}$ such that $f^{m}\left(W^{p}\right)=W^{i}$. The positive integer $m$ is called the preperiod of $W^{p}$.

Definition 6. A path $\left\{W_{t}\right\}_{t=0}^{\infty}$ associated with the real-valued map $f$ : $B \longrightarrow B$ is aperiodic if it is neither periodic, asymptotically periodic, nor preperiodic.

Definition 7. A subset $S$ of $B$ is called invariant under the real-valued map $f: B \longrightarrow B$ if $f(S) \subset S$.

Definition 8. A compact set $A \subset B$ is an attracting set under the realvalued map $f: B \longrightarrow B$ if

(a) $A$ is invariant under $f$.

(b) There exists an open neighborhood $N$ of $A$ such that, for all $x \in N$, $f^{n}(x) \in N$ and $\lim _{n \rightarrow \infty} f^{n}(x) \in A$.

Definition 9. A chaotic attractor $C \subset B$ under the real-valued map $f: B \longrightarrow B$ is an attracting set with positive Lebesgue measure such that 
there exists an invariant set $U \subset C$, with the same Lebesgue measure as $C$, for which the path $\left\{W_{t}\right\}_{t=0}^{\infty}$ associated with $f$ is aperiodic for every initial condition $W_{0} \in U .^{7}$

\section{B Proofs}

Proof of Lemma 3.1. (a) Let us make $e=0$. We see that

$$
\begin{aligned}
W^{M}-W^{m}= & \left(\frac{r \mu}{\xi \rho}\right)^{\frac{1}{\rho-1}}-\left(\frac{A}{r}\right)^{\frac{1}{1-\rho}} \frac{Z}{1+\mu} \rho^{\frac{\rho}{1-\rho}}= \\
& Z\left(\frac{r}{A}\right)^{\frac{1}{\rho-1}}(\rho(1+\mu))^{\frac{\rho}{1-\rho}}\left[\left(\frac{\mu}{\rho}\right)^{\frac{1}{\rho-1}}-(1+\mu)^{\frac{1}{\rho-1}}\right] .
\end{aligned}
$$

Therefore, if $\mu<\frac{\rho}{1-\rho}$, then $W^{m}-W^{M}>0$ and $f$ is a strictly increasing function. Moreover, since $f^{r}\left(W^{m}\right)=f^{l}\left(W^{m}\right)<W^{m}$, we obtain the existence of a unique positive, globally stable fixed point given by

$$
W^{\star}=\left(\frac{1+r \mu(1-\alpha)}{\xi(1-\alpha)}\right)^{\frac{1}{\rho-1}},
$$

with $W^{\star} \in\left(0, W^{m}\right)$.

(b) If $\mu=\frac{\rho}{1-\rho}$, then it is straightforward to see that $W^{M}=W^{m}$ and, as $(1-\alpha) r<1$, we have $W^{*} \in\left(0, W^{M}\right)$. If $\frac{\rho}{1-\rho}<\mu$, then $W^{M}-W^{m}<0$. However, we also have

$$
\begin{gathered}
f^{l}\left(W^{M}\right)-W^{M}=(1-\alpha)\left(W^{M}\right)^{\rho}\left(\xi-r \mu\left(W^{M}\right)^{1-\rho}\right)-W^{M}= \\
W^{M}\left[\frac{(1-\alpha) r(1-\rho) \mu}{\rho}-1\right] .
\end{gathered}
$$

Thus, if $\mu<\frac{\rho}{(1-\alpha) r(1-\rho)}$, we conclude that $f^{l}\left(W^{M}\right)-W^{M}<0$ and, consequently, the unique positive fixed point must satisfy $W^{\star} \in\left(0, W^{M}\right)$.

(c) From the above discussion it is clear that the fixed point lies on the interval $\left[W^{M}, W^{m}\right)$ and $f^{\prime}\left(W^{\star}\right)<0$ under the assumption in the statement of part (c).

Proof of Lemma 3.2. (a) When $e=0$ and $\mu=\frac{\rho}{r(1-\alpha)(1-\rho)}$ the positive stable fixed point corresponds precisely to the maximum of $f^{l}$, that is, $W^{\star}=W^{M}$. Hence, we have $f^{\prime}\left(W^{\star}\right)=0$. As the parameter $\mu$ increases, the derivative at the fixed point becomes negative. Finally, we compute the

\footnotetext{
${ }^{7}$ The complement of $U$ relative to $C$ contains only periodic and preperiodic points.
} 
derivative of $f^{l}$ at $W=W^{\star}$ to obtain $f^{\prime}\left(W^{\star}\right)=(1-\alpha)\left(\frac{\rho}{1-\alpha}-\mu r(1-\rho)\right)$, which is equal to -1 when

$$
\mu=\frac{1+\rho}{r(1-\alpha)(1-\rho)} \equiv \mu_{2}
$$

Therefore, we have a period doubling bifurcation at $\mu=\mu_{2}$ and the stability of the fixed point applies now to the 2-period cycle for $\mu \in\left(\mu_{2}, \mu_{2}+\delta\right)$, with $\delta$ sufficiently small. To finish the proof we must show that the local stability is indeed global. To see this it is enough to show that $f\left(W^{M}\right)-W^{m}<0$ for $\mu \in\left(\frac{\rho}{r(1-\alpha)(1-\rho)}, \mu_{2}+\delta\right)$, or equivalently, that after a finite number of iterates all the dynamics is concentrated in the interval $\left(0, W^{m}\right)$. Obviously, the claim holds for $\mu=\frac{\rho}{r(1-\alpha)(1-\rho)}$, since $f\left(W^{M}\right)=W^{M}<W^{m}$. From the monotonicity of $W^{M}$ and $W^{m}$ with respect to the parameter $\mu$, we only need to check whether the inequality still holds for $\mu=\mu_{2}$. From a direct computation we get

$$
f\left(W^{M}\right)-W^{m}<0 \text { if and only if } r(1-\alpha)(1-\rho) \rho^{\frac{\rho}{1-\rho}}\left(\frac{1+\mu}{\mu^{\rho}}\right)^{\frac{1}{1-\rho}}<1 .
$$

By making $\mu=\mu_{2}$ we get

$$
\begin{aligned}
& r(1-\alpha)(1-\rho) \rho^{\frac{1}{1-\rho}}\left(\frac{1+\mu_{2}}{\mu_{2}^{\rho}}\right)^{\frac{1}{1-\rho}}= \\
& r(1-\alpha)(1-\rho) \rho^{\frac{1}{1-\rho}}\left(\frac{1+\rho}{r(1-\alpha)(1-\rho)}\right)^{\frac{\rho}{\rho-1}}\left(1+\frac{1+\rho}{r(1-\alpha)(1-\rho)}\right)^{\frac{1}{1-\rho}}= \\
& \rho^{\frac{1}{1-\rho}}(1+\rho)^{\frac{\rho}{\rho-1}}(r(1-\alpha)(1-\rho)+1+\rho)^{\frac{1}{1-\rho}}<\left(\frac{2 \rho}{(1+\rho)^{\rho}}\right)^{\frac{1}{1-\rho}}<1
\end{aligned}
$$

for all $\rho \in[0,1)$, which proves parts (a) and (b) in the statement of the Lemma.

Proof of Proposition 3.3. Let us define $\psi=g^{r}$ and $s=0.204$. It is straightforward to see that

$$
\psi^{k}(1)=\frac{-49750}{250 \beta_{\mu}} \sum_{i=0}^{k-1} s^{i}+s^{k}
$$


where $\psi^{k}(1)$ denotes the $k$-iterate of $W=1$ under $\psi .^{8}$ Now we solve the equation $\psi^{k}(1)=W^{m}$ for $\mu$ and we get

$$
\mu_{k}=\frac{\frac{49750\left(\sum_{i=0}^{k-1} s^{i}-250\right)}{12750 s^{k}}+250-25 \sqrt[3]{204}}{25 \sqrt[3]{204}-51} .
$$

Clearly, the sequence $\left\{\mu_{k}\right\}_{k \in N}$ is monotone, tends to infinity and, for each $\mu_{k}$, the associated critical point $W_{\mu_{k}}^{m}$ belongs to a $(k+2)$-period cycle.

P roof of Proposition 3.4. The explicit expression of the fixed point $W^{\star}$ of $g$ as a function of $\mu$ is

$$
W_{\mu}^{\star}=\frac{250\left(-1+250 \sqrt{51}\left(\frac{1+\mu}{250+51 \mu}\right)^{3 / 2}\right)}{\beta(\mu)},
$$

which is a strictly positive, continuous, decreasing function of $\mu$, and tends to 0 as $\mu \rightarrow \infty$. On the other hand, for each $k>0, \psi^{k}(1)$ is an strictly increasing function of $\mu$ tending to $0.204^{k}$, which is bounded away from zero, as $\mu \rightarrow \infty$. Hence, by Bolzano's Theorem, for any $k>0$ there is a unique point $\mu_{k}^{\prime}$ solving for $\mu$ the equation $W_{\mu}^{\star}=\psi^{k}(1)$. Obviously, $\mu_{k}^{\prime} \rightarrow \infty$ as $k \rightarrow \infty$.

Proof of Proposition 4.1. (a) Obvious.

(b) We just have to notice that, if $\mu \in\left[0, \mu^{m}\right)$, the function $f$ is strictly increasing. Moreover, $f(0)=e>0$ and $f^{r}\left(W_{t}\right)$ is a linear function with slope less than 1 . Hence, there exists a $W^{\star}$ such that $f\left(W^{\star}\right)=W^{\star}$, with $0<f^{\prime}\left(W^{\star}\right)<1$.

(c) From the functional form of the function $f$, we see that for sufficiently large values of $\mu$ the function $f$ has local maximum at $W^{M}$ and a local minimum at $W^{m}$ with $W^{M}<W^{m}$. Moreover, it is easy to see from (10) that $W^{m}$ tends to 0 as $\mu$ goes to infinity. Clearly, for a given $e>0$, a sufficient condition for having a globally stable fixed point is $f\left(W^{m}\right) \geq W^{m}$, where the fixed point $W^{\star}$ satisfies $W^{m} \leq W^{\star}$. An easy computation shows that the last inequality is equivalent to

$$
W^{m} \leq \frac{(1-\alpha)}{1-(1-\alpha) r}
$$

which holds for large enough $\mu$, since $W^{m}$ tends to zero as $\mu$ tends to infinity. The solution to the equation $W^{m}=\frac{(1-\alpha)}{1-(1-\alpha) r}$ gives the value of $\mu^{M}(e)$.

Proof of Proposition 4.2. If $f\left(W^{m}\right)>W^{m}$, where $W^{m}$ is given by (10), then the fixed point lies on the linear part of $f$ and it is stable because

\footnotetext{
${ }^{8}$ Notice that we are not iterating $g$ but $\psi \equiv g^{r}$.
} 
$f^{\prime}\left(W^{\star}\right)=(1-\alpha) r<1$ by hypothesis. Some computations show that $f\left(W^{m}\right)>W^{m} \Leftrightarrow(1-\alpha) r W^{m}+(1-\alpha) e>W^{m} \Leftrightarrow$

$$
e>\frac{1-(1-\alpha) r}{1-\alpha} W^{m} \Leftrightarrow e>\frac{1-(1-\alpha) r}{1-\alpha}\left(\frac{A}{r}\right)^{\frac{1}{1-\rho}} \frac{Z}{1+\mu} \rho^{\frac{\rho}{1-\rho}} .
$$

Finally, note that the RHS term in the last inequality is larger than $e^{*}$ when $\mu \geq 0$, which means that $e>e^{*}$. In particular, when $f\left(W^{m}\right) \geq W^{m}$, the fixed point $W^{\star}$ turns out to be equal to $\frac{(1-\alpha) e}{1-(1-\alpha) r}$. 

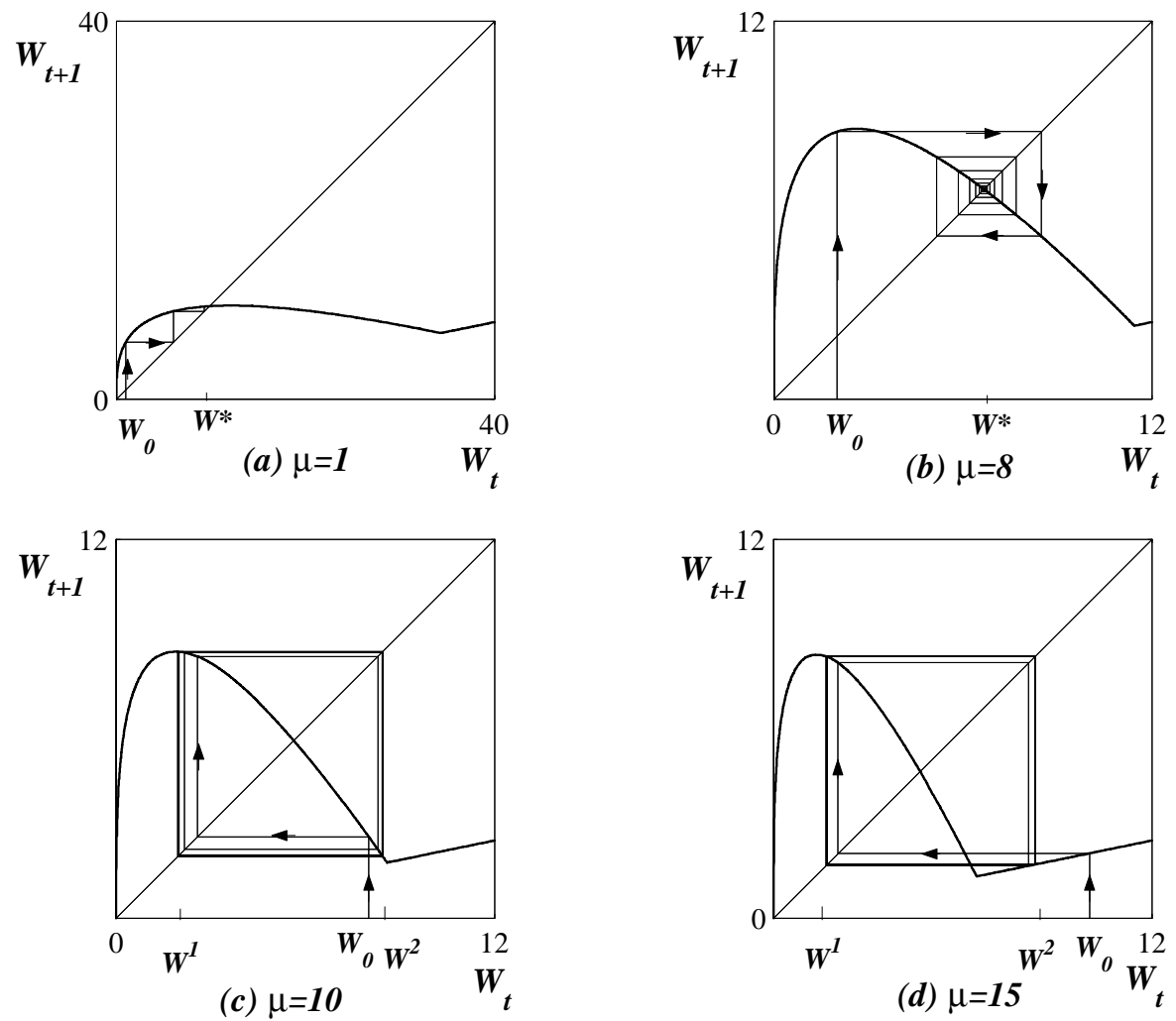

Figure 1: $(a)$ Globally stable fixed point $W^{\star}<W^{M} ;(b)$ globally stable fixed point such that $W^{M}<W^{\star}<W^{m} ;(c)$ period 2 attracting cycle $\left\{W^{1}, W^{2}\right\}$ such that $W^{M}<W^{1}<W^{2}<W^{m} ;(d)$ period 2 attracting cycle $\left\{W^{1}, W^{2}\right\}$ such that $W^{M}<W^{1}<W^{m}<W^{2}$. 


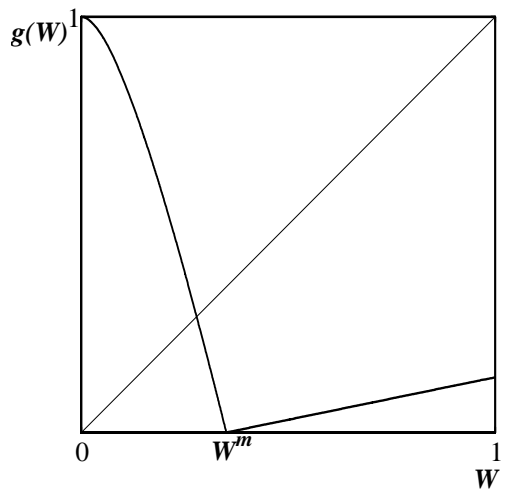

Figure 2: Function $g$ for an arbitrary value of $\mu>25.239$. 

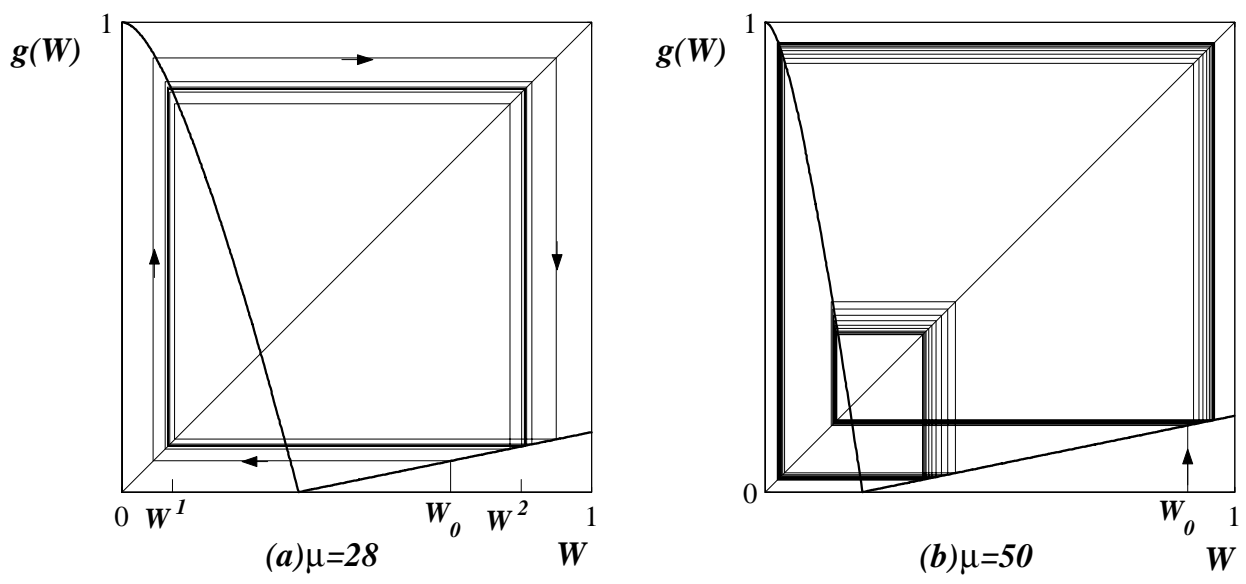

Figure 3: $(a)$ Period 2 attracting cycle, $(b)$ period 4 attracting cycle. 

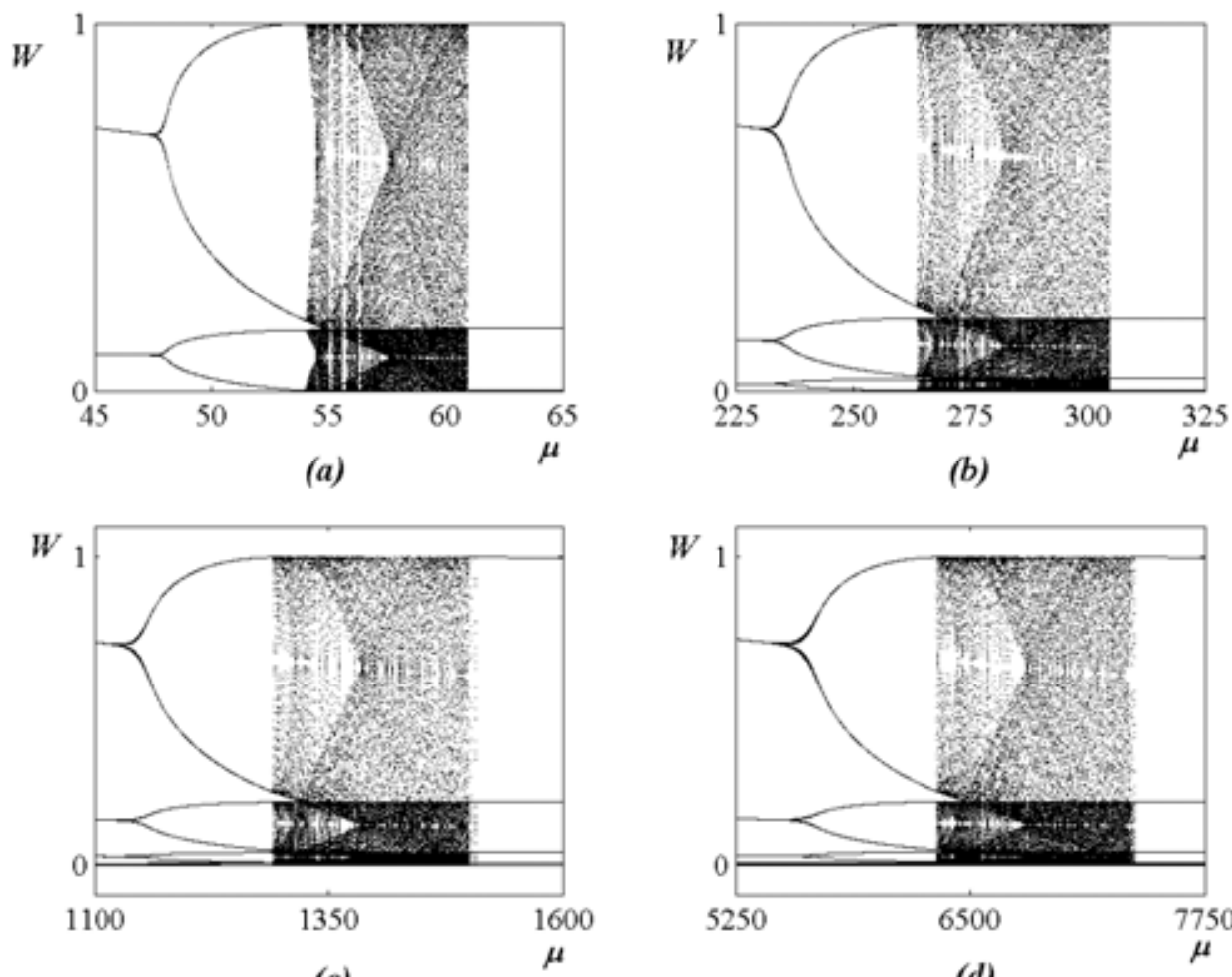

(c)

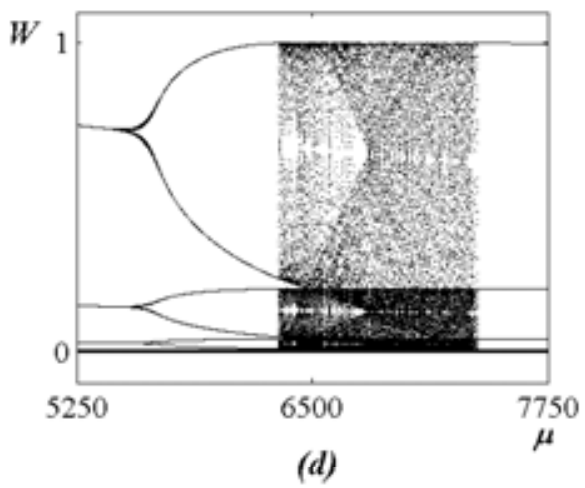

Figure 4: Bifurcation diagrams for different intervals of the parameter $\mu$. 

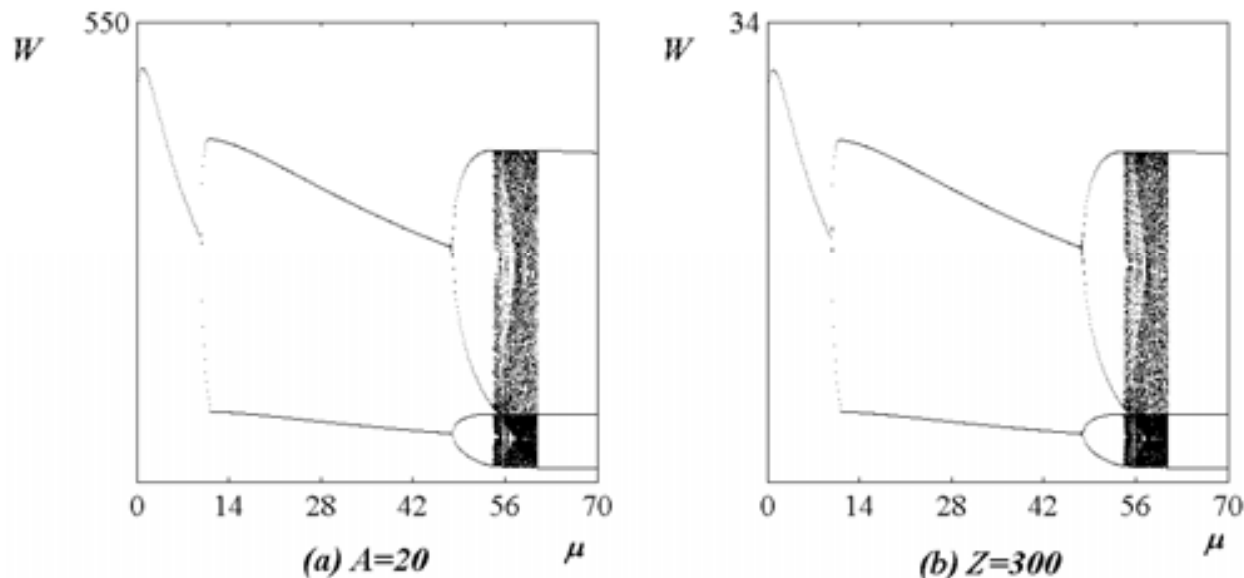

Figure 5: (a) Bifurcation diagram of $f$ for $\mu \in[0,70]$ and $A=20,(b)$ bifurcation diagram of $f$ for $\mu \in[0,70]$ and $Z=300$. 

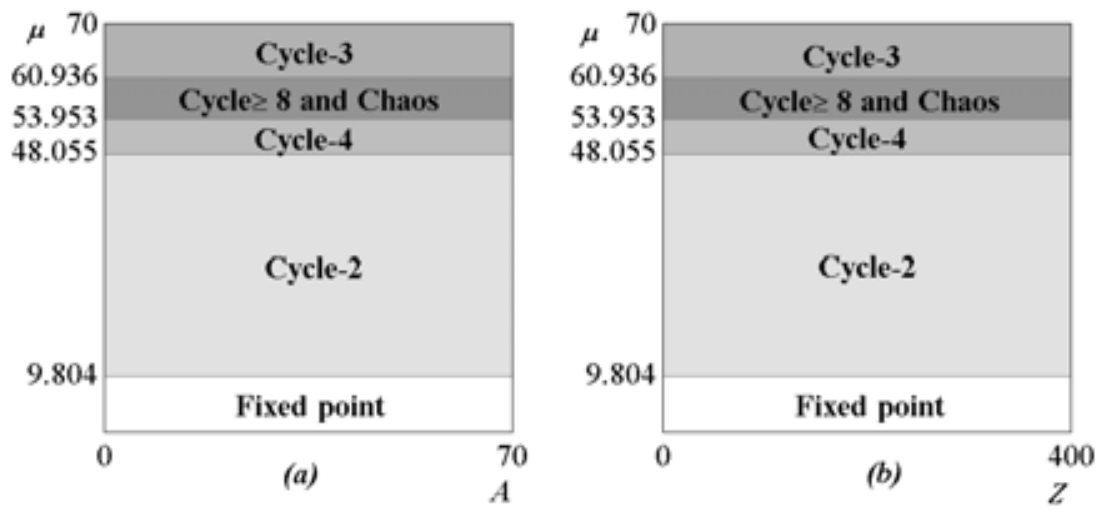

Figure 6: $(a)$ Attractors of $f$ in the parameter plan $(A, \mu),(b)$ attractors of $f$ in the parameter plan $(Z, \mu)$. The bifurcation curves are straight lines. 

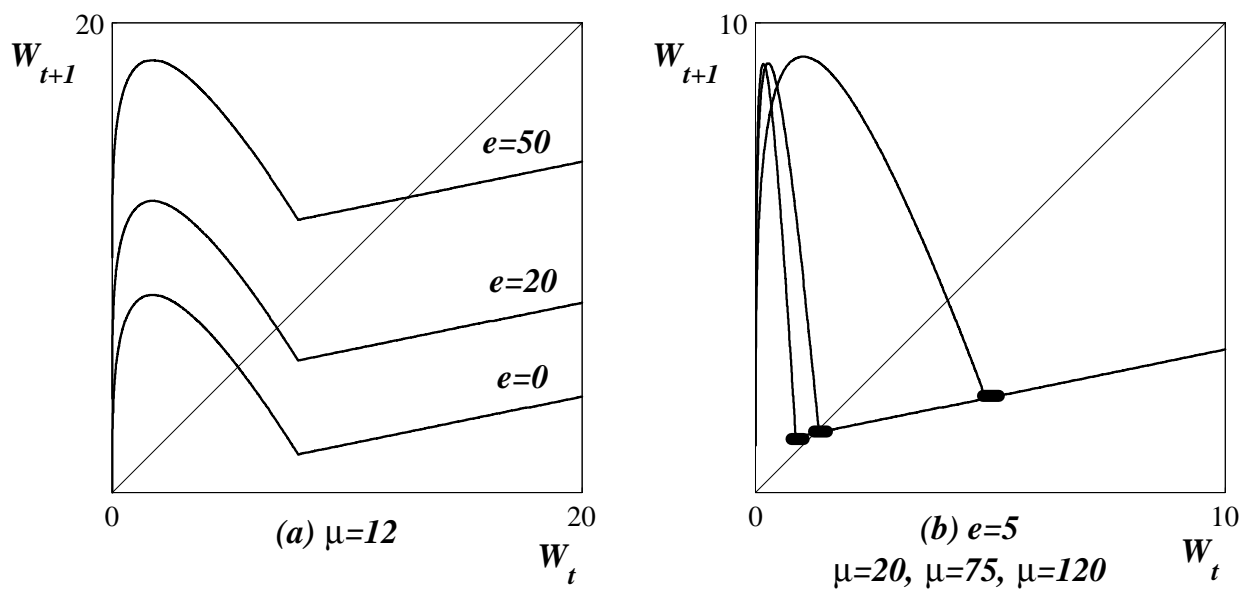

Figure 7: (a) Vertical shift of $f$ for different positive values of $e,(b)$ the minimum point passing from the right to the left of the diagonal when $\mu$ increases. 

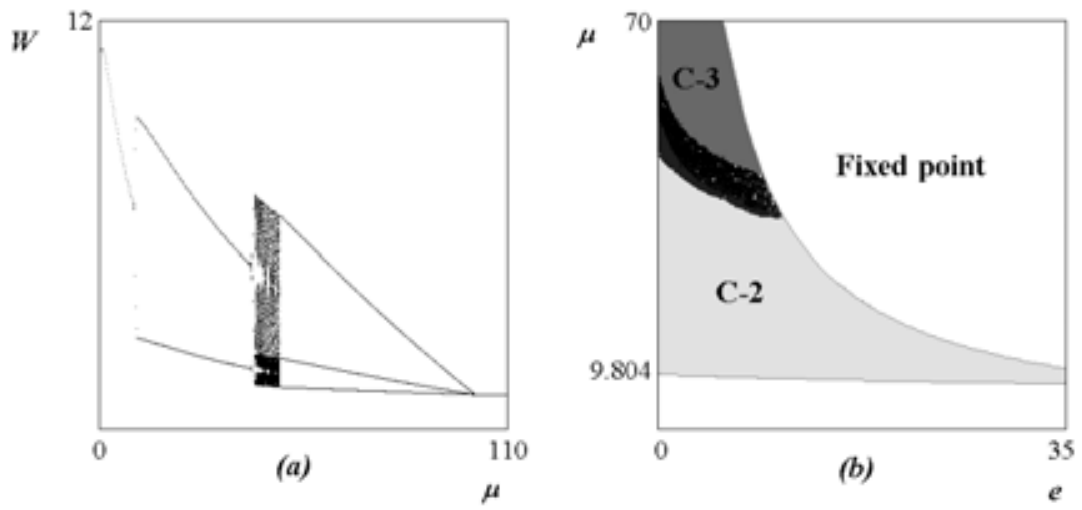

Figure 8: (a) Bifurcation diagram of $f$ with $e=4,(b)$ attractors of $f$ in the parameter plan $(e, \mu)$; the hyperbolic curve is $\mu^{M}(e)$. 